\title{
Questes
}

Revue pluridisciplinaire d'études médiévales

\section{Les fondations pieuses et la construction de la mémoire. Le cas de Jean Rose, bourgeois de Meaux du XIV ${ }^{\mathrm{e}}$ siècle}

\section{Mickaël Wilmart}

\section{(2) OpenEdition \\ 1 Journals}

Édition électronique

URL : http://journals.openedition.org/questes/2608

DOI : 10.4000/questes.2608

ISSN : 2109-9472

Éditeur

Les Amis de Questes

\section{Édition imprimée}

Date de publication : 15 décembre 2002

Pagination : 7-9

ISSN : 2102-7188

\section{Référence électronique}

Mickaël Wilmart, «Les fondations pieuses et la construction de la mémoire. Le cas de Jean Rose,

bourgeois de Meaux du xive siècle ", Questes [En ligne], 2 | 2002, mis en ligne le 01 janvier 2014, consulté le 15 septembre 2020. URL : http://journals.openedition.org/questes/2608 
duc d'un second surnom, dont le prestige efface cette fois la puissance capétienne et le lignage - surnom naturellement inventé de toute pièce par Chastelain lui-même. Ainsi la rhétorique «auguste » de l'historiographe augmente la gloire de l'Auguste duc, en soulignant la singularité d'un prince, unique en son temps. L'écriture se constitue, dans le même mouvement, en source d'elle-même : le lion de Bourgogne, le grand duc d'Occident renvoient, en fait, à l'écriture de Chastelain et participe à sa gloire. Le nom du prince est remplacé par le surnom littéraire dans la mémoire des hommes, signe de la complicité de l'écrivain et de son commanditaire qui caractérise l'œuvre des premiers Rhétoriqueurs.

\section{Mickaël WILMART, « Les fondations pieuses et la construction de la mémoire. Le cas de Jean Rose, bourgeois de Meaux du XIV ${ }^{\mathrm{e}}$ siècle »}

Au delà de l'aspect religieux reflétant la piété du bienfaiteur, doter une église ou un établissement ecclésiastique, fonder une messe ou mieux une institution de charité sont des actions qui s'inscrivent dans un phénomène plus vaste de construction de la mémoire de l'individu. Sa générosité assure, outre le salut de son âme, la postérité d'une bonne réputation. Cette mémoire prend en premier lieu la forme de l'écrit : les obituaires dressent la liste des offices pour les donateurs, conservant ainsi leurs noms que les générations suivantes continueront à honorer pour leurs bienfaits.

Il arrive parfois que la richesse d'un homme l'aide à dépasser la mémoire écrite pour une mémoire de la pierre se dessinant dans le paysage urbain. Ainsi, Jean Rose, bourgeois de Meaux (Seine-et-Marne) mort en 1364, est devenu à travers les siècles le héros de toute une ville. A la mort de son père, Jean Rose hérite de la plus grande fortune de Meaux. Au XIII ${ }^{\mathrm{e}}$ siècle, ses ancêtres étaient des drapiers et c'est sans doute dans cette activité qu'il faut en chercher l'origine. Cette richesse, apparemment largement supérieure à celles de ses concitoyens, permet à Jean Rose de faire de nombreuses fondations pieuses. Surtout, elle va faire de lui un bâtisseur.

Son épouse Jeanne meurt en 1328. A partir de cette date, il accumule des biens en vue de fonder une chapelle dans la cathédrale. Alors que dans la plupart des villes, les bourgeois fondent l'office de la chapelle ou/et financent les ornements ou les vitraux, Jean Rose donne l'argent nécessaire à sa construction. En 1331, la nouvelle chapelle collatérale de la cathédrale est achevée et on y installe l'autel du SaintSacrement. Cette installation n'est sans doute pas anodine dans la construction d'une mémoire si on prend en considération l'importance de la dévotion des fidèles au Saint-Sacrement. Or, Jean Rose fait de cette chapelle «sa» chapelle. Dans un premier temps il y place la sépulture de son épouse décédée et y prévoit la sienne. A cette fin, on réalise la pierre tombale du couple, toujours conservée dans la cathédrale, qui fait apparaître un raffinement supérieur à celui des tombes ecclésiastiques du sanctuaire. La dalle se caractérise par sa polychromie composée d'un calcaire carbonifère noir incrusté de marbre blanc et d'un mastic coloré dessinant 
le relief des vêtements. L'analyse de ces divers éléments a conduit les historiens de l'art à rattacher cette tombe aux ateliers de Tournai. L'empreinte de Jean Rose sur la chapelle est renforcée par la réalisation d'une fresque (encore visible au XIX ${ }^{\mathrm{e}}$ siècle) le représentant avec sa femme sur l'un des murs. Aussi, le souvenir de Jean Rose frappait le fidèle à chaque fois qu'il passait devant la chapelle du Saint-Sacrement.

Cette présence dans le décor intérieur de la cathédrale va se doubler d'une autre construction, près de la porte orientale de la ville (une des plus passantes) : l'hôpital de la Passion fondé par Jean Rose en 1356. A cette date, il fait édifier un établissement permettant l'accueil de vingt-cinq aveugles et de douze pauvres. Il y ajoute une école pour dix enfants pauvres de la ville « habiles à apprendre ». Dès lors, cet hôpital et Jean Rose ne font qu'un dans l'imaginaire meldois. L'amalgame trouve d'abord son origine dans les actes touchant les biens de l'établissement. Quand un bien lui est vendu, il l'est «à Jean Rose et aux pauvres de l'hôpital de la Passion », montrant le soutien financier du bourgeois. Quand il s'agit d'une donation, elle est faite «à l'hôpital de la Passion fondé par Jean Rose (ou feu Jean Rose après son décès) ». L'entourage du fondateur fait rapidement de la chapelle de l'hôpital son sanctuaire. Dans un premier temps, son fils, avocat au Parlement de Paris, s'y fait inhumé. Puis vient Guillaume de Marchières en 1376 qui demande à élire sa «sepulture ou moustier dudit hospital pres de feu vaillant homme et sage maistre Jehan Rose fil dudit fondeur ». Auparavant, il a rappelé dans son testament sa dévotion « en l'onneur et remembrance de la tres glorieuse Passion de notre tres douls glorieuls dieu Iehu Crist, de laquelle noble Passion feu vaillant homme et sage de bonne memoire sire Jehan Rose jadis bourgeois de Meaulx de son temps à l'aide la Sainte Trinité fonda l'ospital seant à Meaulx lès la porte Saint Remy ». En 1415, Simon Rose, petit-fils du fondateur se fait également enterrer dans la chapelle. Quelques années plus tard, lors de la rénovation de celle-ci, on place les armes de Jean Rose au dessus du portail donnant sur la rue. Enfin, dans les dernières décennies $\mathrm{du} \mathrm{XV}^{\mathrm{e}}$ siècle et au cours du $\mathrm{XVI}^{\mathrm{e}}$ siècle, l'hôpital de la Passion change progressivement de nom, devenant pour tous l'hôpital Jean Rose.

L'historiographie de la ville du $\mathrm{XVI}^{\mathrm{e}}$ au $\mathrm{XX}^{\mathrm{e}}$ siècle se charge alors de finir de construire la mémoire d'un «bienfaiteur de Meaux » en ne retenant que la fondation de cet hôpital, pourtant le quatrième établissement du genre dans la cité après le Grand Hôtel-Dieu, la léproserie Saint-Lazare et l'hôtel-Dieu Cornillon. Sa qualité de bourgeois fait sans aucun doute de Jean Rose le symbole d'une bourgeoisie locale prospère et surtout charitable. En 1819, Pierre Navarre, ancien maire, écrit dans son Essai historique sur la ville de Meaux :

Sous le règne de Jean, fils de Philippe de Valois, la ville de Meaux offroit un homme remarquable par sa philantropie et le pieux usage de ses richesses. Jean Roze, suivant la tradition, en avoit acquis de considérables, en achetant des blés au-dessus du cours, lorsqu'ils étoient chers ; de manière que ses greniers étoient comme autant de magasins pour les temps de disette. Aussi, loin que le commerce de cette denrée ait terni sa mémoire, elle est encore aujourd'hui en vénération à Meaux, comme celle d'un bienfaiteur de cette ville. Entre autres oeuvres pies, il y a fondé un hôpital, longtemps appelé de son nom. 
Cette tradition de l'origine philanthropique de la fortune de Jean Rose est alors récente (les historiographes du XVIII ${ }^{\mathrm{e}}$ siècle n'en parlent pas) et n'est surtout fondée sur aucun document. Elle est le fruit de l'amalgame de Jean Rose, plus ancien bourgeois connu de tous et fondateur d'un hôpital, et de Nicolas Tronchon qui sauva la ville de Meaux de la disette en 1789 en ouvrant ses greniers et vendant son grain à bon prix. Nicolas Tronchon était alors devenu le symbole de la solidarité et fut décoré par la municipalité. Il fallait trouver à cette générosité de l'élite briarde une ancienneté remontant à des temps immémoriaux. Le fondateur d'un hôpital au Moyen Age ne pouvait-il pas jouer ce rôle ? La bourgeoisie du XIX ${ }^{\mathrm{e}}$ siècle fait donc de cet « ancêtre » son héros. Après avoir envisagé de lui ériger une statue, la municipalité donne son nom, en 1846, à l'une des artères de la ville. Les érudits des sociétés savantes naissantes, toujours sans consulter des archives pourtant existantes, se chargent à leur tour d'entretenir sa mémoire en insistant uniquement sur sa générosité. Dans les années 1930, le journal Le Courrier de Seine-et-Marne crée une rubrique rapportant les opinions du meldois moyen intitulée «Les propos du bonhomme Jean Rose » assimilant l'ensemble de la population de Meaux à son bienfaiteur. Dans le même temps, sa pierre tombale est redressée et scellée au mur de la chapelle fondée en 1331 pour assurer sa conservation. Les quelques autres dalles médiévales sont remisées dans les réserves du musée municipal. Seule celle de Jean Rose suscite encore l'admiration des visiteurs, sous les commentaires bienveillants qui perpétuent la tradition d'une mémoire reconstruite à travers les siècles.

\section{Nelly Labère, «La fresche mémoire : génération et régénération dans les Cent Nouvelles Nouvelles »}

Les Cent Nouvelles Nouvelles constituent le premier recueil de langue française à revendiquer le terme de «nouvelles » pour qualifier leur production narrative. Mais cette primauté ne va pas sans un jeu sur le temps et la mémoire, y compris sur le mode de la rupture. En effet, employer le terme de «nouvelle» n'est pas sans implications.

Le substantif «nouvelle » inscrit en creux la figure de Boccace et dessine un rapport de filiation complexe à travers une mémoire auctoriale. Ce sont bien des Cent Nouvelles mais des Cent Nouvelles nouvelles. De l'imitation à l'écart, le jeu référentiel induit des choix narratifs qui peuvent être lourds de sens. En effet, le recueil bourguignon procède à une modification de poids : il opère le passage entre le terme «décaméron» et celui de «cent nouvelles» pour désigner une production narrative. Les «cent nouvelles» du contenu boccacien deviennent ainsi dans le recueil bourguignon un marquage générique. Cette transformation d'un recueil à l'autre engage des enjeux esthétiques qui dépasseraient le simple jeu sur l'attestation référentielle et contiendraient les germes d'un nouvel art poétique. Celui-ci se fonderait alors sur une exigence : la problématique alliance de la nouvelle et de la nouveauté. 